\title{
ANTROPOLOGIA PELA EDUCAÇÃO. NOTAS POR UMA DESCOLONIZAÇÃO DO PENSAMENTO*
}

\author{
Roberto Malighetti ${ }^{* *}$
}

\begin{abstract}
RESUMO: $\mathrm{O}$ artigo analisa comparativamente os mecanismos do pensamento colonial e as suas resistências nas políticas públicas contemporâneas através das fragmentarias ideologias e práticas multiculturalistas. Mostra como agindo seletivamente sobre os mecanismos indenitários, promovem açóes especiais e emergenciais que superam o Direito e fagocitam as contradiçôes políticas e econômicas estruturais. Discute a centralidade dos grupos marginais para ultrapassar as epistemes dicotômicas de matriz colonial (identidade/alteridade, homogeneidade/diferença, hegemonia/subalternidade, centro/periferia, desenvolvimento/subdesenvolvimento). Convida a individuar como os povos colonizados e escravizados, migrantes e refugiados, prófugos e clandestinos, indígenas e indigentes possam articular a superação das dramáticas desigualdades socioeconômicas e da aquisição formal de direitos já definidos com a redefinição e o reconhecimento de novos direitos.
\end{abstract}

Palavras-chave: Descolonização. Identidade. Violência. Multiculturalismo. Cidadania.

\section{ANTHROPOLOGY FOR EDUCATION: FOR A DECOLONIZATION OF THOUGHT}

ABSTRACT: The article makes a comparative analysis of the colonial thought mechanisms and their resistance in the contemporary public policies through the fragmentary ideologies and multicultural practices. It shows how, by acting selectively over identitary mechanisms, they promote special and emergency actions which disregard the Law and feed upon the political and economical structural contradictions. It discusses the centrality of marginal groups in overcoming the dichotomic episteme of a colonial nature (identity/ alterity, homogeneity/difference, hegemony/subalterneity, centre/periphery, development/ underdevelopment). It invites us to describe how the colonized and enslaved, migrants and refugees, fugitives and clandestines, indigenous people and indigents can articulate the act of overcoming the dramatic

\footnotetext{
* Artigo escrito originalmente em português com revisão técnica de Sebastião Moreira Duarte (UFMA).

** Università degli Studi di Milano-Bicocca, Dipartimento di Scienze Umane per la Formazione “Riccardo Massa”, Milano, Italia.E-mail de contato: roberto.malighetti@unimib.it.
} 
socioeconomical inequalities and the formal acquisition of rights already defined, with the redefinition and acknowledgement of new rights.

Keywords: Decolonization. Identity. Violence. Multiculturalism. Citizenship.

\section{ANTHROPOLOGIE DE L'ÉDUCATION: POUR UNE DÉCOLONISATION DE LA PENSÉE}

RÉSUMÉ: The article makes a comparative analysis of the colonial thought mechanisms and their resistance in the contemporary public policies through the fragmentary ideologies and multicultural practices. It shows how, by acting selectively over identitary mechanisms, they promote special and emergency actions which disregard the Law and feed upon the political and economical structural contradictions. It discusses the centrality of marginal groups in overcoming the dichotomic episteme of a colonial nature (identity/ alterity, homogeneity/difference, hegemony/subalterneity, centre/periphery, development/ underdevelopment). It invites us to describe how the colonized and enslaved, migrants and refugees, fugitives and clandestines, indigenous people and indigents can articulate the act of overcoming the dramatic socioeconomical inequalities and the formal acquisition of rights already defined, with the redefinition and acknowledgement of new rights.

Mots-clés: La décolonisation. L'identité. La violence. Le multiculturalisme. La citoyenneté.

The Roman Divide et impera was the great rule by which Great Britain [...] contrived to retain the tenure of her Indian empire. The antagonism of the various races, tribes, castes, creeds and sovereignties, the aggregate of which forms the geographical unity of what is called India, continued to be the vital principle of British supremacy.

(Karl Marx, New York Daily Tribune, July 15, 1857).

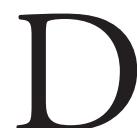

ivide et impera. O famoso dito de Caius Julius Caesar constitui o princípio originário e fundador do pensamento colonial. Exprime as ideologias e as práticas de domínio articulando a fragmentação dos componentes sociais com a integração vertical da sociedade. Através da emissão seletiva de privilégios realiza a homogeneização das contradições e a neutralização das possibilidades de coalizões e oposições.

As políticas coloniais marcaram a história da humanidade. No século passado valeram-se do suporte da Antropologia Social, utilizando a congruência entre a análise funcionalista dos sistemas sociais indígenas com os modelos do duplo mandato (dual mandate) e do governo indireto (indirect rule). (MALIGHETTI, 
2001) Priorizando os topoi constitutivos da ciência da cultura (comunidade, identidade, etnia, tribo, nação) como conjuntos orgânicos de partes integradas e discretas, os estados coloniais organizaram e governaram os grupos sociais: inventaram as tribos na África sobre a base do modelo europeu do Estado-Nação (SOUTHALL, 1970), colocando-as umas contra as outras mediante concessões discriminatórias de direitos especiais.

$\mathrm{Na}$ contemporaneidade, as ideologias e as práticas multiculturalistas renovam a estrutura estratégica inaugurada pelo Senado romano. Pensando a sociedade como um mosaico de monoculturas minoritárias homogêneas com fronteiras bem definidas, as políticas multiculturalistas essencializam a mútua distinção e a coerência interna, construída, alternativamente, acerca de variáveis culturais, genealógicas, territoriais, religiosas, linguísticas ou raciais. Combinam a integração social com a organização vertical das estratificaçôes socioeconômicas internas e excluem as articulações das diferenças segundo óticas complexas (classe, gênero, status, idade etc.). As diversidades são institucionalizadas nos termos unívocos dos nichos criados pelas administraçóes: a identidade cultural única e uniforme é o pré-requisito fundamental para o reconhecimento social e político e para o eventual acesso à cidadania.

\section{Violências do idem}

$\mathrm{O}$ uso da identidade como tecnologia de poder entrega os potenciais antagonismos e os conflitos sociais ao domínio centralizado do Estado, reproduzindo os dispositivos do racismo analisados por Foucault (1976): as fragmentações identitárias, assim como as hierarquias raciais, revelam-se instrumentos do biopoder para exercer uma soberania eugênica sobre a ordem social, contrastando as ameaças dos fatores exógenos e endógenos, a mudança e as contradiçóes socioeconômicas. A fundação dos grupos com base nos sentimentos primordiais de pertencimento e na naturalização do próprio critério de diferenciação é estruturalmente ligada ao domínio e ao controle social e a formação de lealdades contra os elementos antagônicos.

A fenomenologia da identidade, na sua forma constitutiva, assume, maquiavelicamente, as figuras do fechamento e da ameaça (REMOTTI, 2010) e envolve diversos métodos catárticos que purificam da sujeira interna e da contaminação externa (DOUGLAS, 1966), negando as ligaçóes, as diversidades estruturais e o fluxo temporal. A afirmação de um núcleo substancial subtrai os direitos, os privilégios, as prerrogativas, as conquistas, o território do idem, das possibilidades de alteração. Ao mesmo tempo, a construção do alter, como ameaça, torna insuportável qualquer alteração da integridade. As minorias, os seres humanos "insuficientes", os desviantes, são considerados subversivos da relação que coloca 
em recíproca definição pureza, totalidade e fronteira social inviolada, e tornam-se os primeiros objetivos das marginalizações e das depuraçôes. (MALIGHETTI, 2007)

A fragilidade estrutural da identidade leva as políticas a passarem da defesa de um pluralismo neutro e objetivo para formas de agressão avessas a contaminaçóes. O gérmen do genocídio e da "solução final" do outro é a conclusão contida, desde o início, na dialética da identidade. Fundada sobre mapas necrográficos e retóricas pseudo-demográficas, reage a ameaças procurando eliminar a "sujeira" que as separa da pureza. (APPADURAI, 2005; REMOTTI, 2010)

A limpeza étnica atravessou a história da humanidade assumindo, como processo de uniformização, o ato fundador das realidades estaduais. (BAUMANN, 1996) Diversas perspectivas consideram a violência como um ingrediente da identidade, expresso nas tentativas de impor os modelos ideais da uniformidade sobre realidades sociais e estruturais variadas e em constante alteração. A violência é uma técnica para "imaginar uma comunidade" (ANDERSON, 1983), um modo de produzir a "adesão total" (APPADURAI, 1998) ou uma "pratica de constituição da comunidade”. (GOUREVITCH, 1998, p. 95) Permite identificar como concretas as categorias étnicas abstratas, atribuídas tanto a um "nós" a preservar, quanto a um "outro" a pseudo-especial (ERIKSON, 1966) subtraindo a sua humanidade, ou a presentificar através de mutilaçóes físicas e corporais, signo tangível da negação da sua presença. (HAYDEN, 1996)

A violência identitária atravessa não só as práticas coloniais e o "populismo autoritário" (HALL, 1985) que junta patriotismo, xenofobia, etnicismo e militarismo como último recurso para sustentar a identificação com o poder e para construir lealdades incondicionais contra as contaminaçóes. A tecnologia da identidade permeia também as políticas pós-coloniais e as reivindicaçóes progressistas de vários movimentos contemporâneos.

Os idiomas dos protagonistas das liberaçóes e dos líderes da pós-independência renovam - como avisou Franz Fanon a propósito dos perigos inerentes ao fetichismo das identidades petrificadas - antigas formas de opressão colonial. (SAID, 1993, p. 295-309) As políticas dos novos estados combinam os irrealizáveis projetos de modernização com imposiçóes de identidades primordiais, baseadas sobre uma mimicry (BHABHA, 1994) que reproduz as rígidas estruturas binárias do pensamento colonial e suas práticas de marginalização. $\mathrm{Na}$ África, o nacionalismo, apoiado por uma intelligentsia mediadora do comércio cultural com a Europa (APPIAH, 1991), utilizou a redução da negritude a uma expressão biológica. A manipulação da ontologia primordialista de Senghor, preferida às complexas e dinâmicas concepçóes de Césaire e Damas, funcionou para esconder e reprimir os conflitos internos e as discriminaçóes. 
Por outro lado, as hodiernas açóes multiculturalistas implementam os ideais verticais e fragmentários de integração nacional através das políticas do reconhecimento em suas diversas configuraçóes. (TAYLOR, 1994) A densa rede de leis "especiais", "transitórias" e "excepcionais" para as minorias, fundada no pressuposto de que o direito para esses grupos é específico e separado dos problemas dos cidadãos do Estado, projeta parte da sociedade para fora dos conceitos de nação e de comunidade em termos coerentes com formas administrativa de "inclusão excludente". (AGAMBEN, 1995) Tais políticas fagocitam as lutas pelo reconhecimento dos direitos e contrastam a deslegitimação do Estado-Nação e a erosão da hegemonia das classes dominantes, agindo seletivamente sobre os mecanismos endógeno e halógeno, mistificando as profundas disparidades estruturais.

As diferentes conformaçóes daquilo que Spivak (1999) define como essencialismo estratégico, as diversas "ações afirmativas" ou as "discriminaçôes positivas", coludem com as lógicas reacionárias, sustentando-as. Incluídas em um quadro fenomenológico como "erro necessário" para conseguir o reconhecimento de direitos civis e políticos, em vez de criar um cavalo de Troia progressista apto a abrir espaços para a participação política e civil, realizam um congelamento metonímico (APPADURAI, 1996) que circunscreve e isola as identidades dos indivíduos e dos grupos aos espaços a que são constringidos por poderes alógenos. Defendendo a sedução nativista em uma suposta pureza racial ou étnica, natural e originária, alimentam formas de racismo diferencial (TAGUIEFF, 1988), de absolutismo étnico (GILROY, 1993) ou de identidade tribal (CLIFFORD, 1997) que legitimam o uso de categorias políticas e disciplinares - como a raça e as ideologias raciais - negadas pela história antes que pela ciência. Sobretudo, não alcançam os objetivos de combater as injustiças sociais e reforçar os sujeitos mais fracos, pelos quais estáo dispostos a correr perigos muito graves e restituir a um obscuro passado o pensamento e as ciências antropológicas e sociais. Pelo contrário, sustentam formas de subordinação e de exclusão organizadas e contidas dentro dos dispositivos verticais que neutralizam as possibilidades de lutas e solidariedades horizontais entre as diferentes categorias de excluídos.

$\mathrm{Na}$ Índia, as quotas no setor público e nas universidades têm beneficiado as castas identificadas pelo governo como "inferiores" em detrimento de outros cidadãos marginalizados. Na África do Sul, a abstrata discriminação positiva e as políticas BEE (Broad-Based Black Economic Empowerment Act), não tiveram impacto sobre as formas de redistribuição de riqueza. Nos Estados Unidos, a discriminação positiva que privilegia os pertencimentos raciais em detrimento da pobreza em geral, além de opor-se ao sonho de Martin Luther King de um futuro "indiferente à cor da pele", demonstra-se coerente com a política que desestimula a igualdade e mais justiça social. Na Europa, a tentativa do Estado de realizar serviços desiguais mobiliza o interesse contra os efeitos e não contra as causas, servindo para evitar uma séria reconsideração da ordem social discriminatória. 


\section{Panem et circenses}

As leis excepcionais e os serviços emergenciais promovem açóes que governam as contraposiçóes entre os grupos que institucionalizam. $\mathrm{O}$ poder de proclamar formas jurídicas excepcionais ou transitórias, como também a suspensão legal da validade das leis ordinárias, exercitam um domínio totalitário, sem qualquer mediação. (SCHMIDT, 1922; BENJAMIN, 1955; AGAMBEN, 1995)

Em nome da segurança, da assistência social ou dos direitos humanos, o estado de emergência autoriza poderes enormes aos executivos, promovendo uma soberania autoritária. $\mathrm{O}$ recurso recorrente a meios extraordinários e a derrogação temporária e contextual acaba com estandardizar-se e tornar-se uma modalidade consuetudinária do contrato social. A inversão da relação entre regra e emergência produz um efeito perverso de continuidade e onipresença da emergência, em consonância com as estratégias das forças dominantes que podem aproveitar-se da universalização desse estado.

A estrutura topológica do estado de exceção é uma técnica de governo paradigmática enquanto geradora da ordem jurídica. É uma figura relacional que não se funda simplesmente na alteridade com respeito às normas jurídicas, mas no "ser tomado de fora", "estar fora e mesmo assim pertencer" e, portanto, incluído através da sua própria exclusão. (AGAMBEN, 2003) Consiste num mecanismo que exclui mantendo a relação com a norma na forma da sua suspensão: não é a exceção que se subtrai à regra, mas a regra que, interrompendo-se, gera a exceçáo e somente assim se constitui enquanto regra. (AGAMBEN, 1995) Esta, deixando de se aplicar, compreende o que a excede e, ao mesmo tempo, cria e define o espaço no qual a ordem jurídico-política assume valor discriminatório e conservador das ideologias e das práticas de marginalização integrativa.

O aparato da exceção modela as políticas de integração social parcial e assimétrica e promove perfis de cidadania limitada, que, no caso brasileiro, som diferentemente nomeadas: geometria variável (LAUTIER, 1997), regulada (SANTOS, 1979), concedida (SALES, 1994), passiva (WERNECK VIANNA, 1997), negativa (CARVALHO, 1991), relacional (DAMATTA, 1991), ou partida. (VENTURA, 1994) Estas conceptualizações de integrações excepcionais manipulam os dualismos simples (dentro-fora, centro-periferia, global-local, norma-desvio) e caracterizam a democracia em termos que combinem "políticas disjuntivas" (HOLSTON, CALDEIRA, 1998), com o exercício de um "controle negociado" (MACHADO DA SILVA, 2002), centrado na subordinação e na dependência.

Os dispositivos excepcionais geram uma forma de exclusão que se materializa nos espaços especiais: não só nos campos analisados por Hannah Arendt (1951) mas também nas comunidades discriminadas de refugiados, de imigrantes, de clandestinos, de vítimas e prisioneiros de guerra, de homens e mulheres trafi- 
cados, de reservas indígenas e de favelas. (MALIGHETTI, 2011) Esses lugares materializam as possibilidades de normalização da exceção. Em tais recintos, delimitados por cercas territoriais e simbólicas, o sistema legal, através de sua suspensão excepcional, inclui e controla o que exclui. Determina a desistoricização e despolitização da realidade dramática em que uma grande parte da população vive, e garante uma ordem eficaz ao conter as possibilidades de reação à condição de pobreza e privação.

Sob a pressão da urgência, as ações se fixam como não-negociáveis, neutralizando o potencial de inovação local. Os problemas sociais são transfigurados em questôes técnicas e emergenciais, inaugurando modelos organizacionais que baseiam a sua legitimidade na performatividade e na eficácia, de maneira totalizante, em detrimento dos modos alternativos de intervenção. As reflexôes críticas sobre as contradiçóes ou as causas da marginalização ou da exclusão são eliminadas. Os fatores desagregantes são, no máximo, considerados em termos apolíticos, mecânicos e naturais, ligados a estados endêmicos naturais, warfare tribais, ou a uma história significativamente considerada local e nunca global.

Agindo como "máquinas antipolíticas" (FERGUSSON, 1990), as configuraçóes especiais suspendem o "político", alimentando o fatalismo, o clientelismo, o assistencialismo e a dependência. $\mathrm{O}$ uso frequente de logística e dos mecanismos que garantem a ordem, a estabilidade e a segurança, estendem o que Giorgio Agamben (1995) chama de "zona cinzenta" de operaçóes militares justificadas como operações humanitárias, nas quais os atores civis têm cada vez menos espaço para autonomia e liberdade.

Em nome da emergência vários dispositivos e técnicas de subjetivação (associações, centros de aceitação, tribunais, igrejas, serviços sociais, escolas, sociedades, ONGs) operam no território como aquilo que Appadurai (1996) chama de "formas móveis de soberania", realidades que se deslocam impondo regras e imperativos legitimados sob a bandeira de valores indiscutíveis. "Especialistas da subjetividade" (ROUSE, 1995) nacionais ou internacionais, exercem poder e soberania, adaptando e redefinindo as práticas de governabilidade. "Modernizadores intermédios" (RABINOW, 2003) desagregam as redes de influência, modificam os sistemas de poder preexistentes, estendem novas alianças e confundem as estratégias das autoridades locais: designam as competências, distribuem funçôes, integram grupos locais nos circuitos nacionais e internacionais como novas formas de governance. Uma pletora de gerentes, burocratas, cientistas, técnicos, voluntários, forçam a participar (ou, melhor, "a ser participados") só em termos de identidade artificialmente produzidos pelos idiomas e pelas categorias dos projetos. Estes programas constroem os interlocutores e os grupos sociais segundo modelos integrados e visões estereotipadas que, escondendo a seleção dos beneficiários necessariamente exclusiva, enfatizam a homogeneidade orgânica e ignoram a estratificação de poder que determina o controle e o uso dos recursos. 
A dimensão biopolítica do estado de exceção destaca os quadros jurídicos e políticos das relaçóes entre o Estado e os indivíduos em termos paradoxais. Embora a lei clássica pense em termos de indivíduos e de sociedade, de cidadãos e de Estado, o aparato especial raciocina em termos de entidades abstratas, destinadas a serem identificadas, pesquisadas, recenseadas, registradas, contadas, quantificadas, catalogadas, etnicizadas. A estratégias e as categorias diagnósticas das administraçôes, exportáveis em todos os contextos, reduzem a identificação dos cidadãos à biologia, à "abstrata nudez do ser unicamente humano e nada mais" (ARENDT, 2000 [1951] p. 327) ou à “vida nua”. (AGAMBEN, 2003)

\section{Exceder as exceções}

Nos ecúmenos complexos e globais da contemporaneidade, os atores sociais tradicionalmente percebidos através das categorias da marginalidade procuram escapar às técnicas de governabilidade e às práticas de normação. Várias experiências subalternas contradizem a pax romana das tentativas de promover uma ideologia multiculturalista feliz e pacificada.

Povos colonizados e escravizados, migrantes e refugiados, exilados e clandestinos, indígenas e indigentes, rejeitam fundaçóes em termos absolutistas, configurando-se, contingentemente e precariamente, como redes que envolvem vários posicionamentos. Estes protagonistas desmantelam os sistemas de classificação, desconstruindo e esvaziando as identidades e superando a lógica dicotômica colonialista conotada em termos biológico-naturais. Novas formas de subjetividade abrem espaços antropoiéticos (REMOTTI, 2010) que transcendem as reificações das diferenças. Mostram que cada cultura tem sido sempre híbrida (CANCLINI, 1990), produto de uma longa história de apropriações, resistências, antagonismos, incoerências, contradições. As culturas não são complexas ou "loucas" (CLIFFORD, 1988) apenas no mundo contemporâneo, por causa da globalização, que, de qualquer forma, não é uma dinâmica recente, tendo constituído a história da humanidade, desde quando os primeiros afrodescendentes deixaram a África Oriental 70 mil anos atrás para povoar o planeta. (AMSELLE, 2001)

Laboratórios de formas de humanidade exibem uma dinâmica baseada na fusão, na mistura e na oposição. As suas práticas superam a substituição do local pelo global ou do tradicional pelo moderno, em favor de concepçóes de globalidades ou modernidades "múltiplas" (COMAROFF, 1993), entendidas como conjuntos de realidades negociais produzidas pelo co-pertencimento (GADAMER, 1965) da modernidade e da tradição do global e do local. O Atlântico Negro é a metáfora dos reconhecimentos culturais, políticos e estéticos irredutíveis à qualquer essencialização identitária. (GILROY, 1993) As suas travelling cultures são produzidas pelos confrontos, encontros, viagens, fusóes e resistências. 
Enquanto formas de criatividades culturais subtraem o global da universalidade abstrata imposta pelas ideologias dominantes, colocando-o nas articulaçóes dos microprocessos cotidianos, microglobais ou micro-modernos, necessariamente locais e particulares.

Atravessados por diferentes origens culturais e por uma multiplicidade de posicionamentos, os atores transnacionais cruzam e expandem as fronteiras, não se configurando de acordo com as lógicas sedentárias ou com o ordenamento dualístico (nacional-extranacional, interior-exterior autóctone-estrangeiro). Estas "presenças-ausentes" (SAYAD, 1991) negociam tipos de pertencimento múltiplo e de adaptaçóes práticas e técnicas com à flexibilidade do posicionamento geográfico e social, efeito das novas articulações entre as várias comunidades políticas e econômicas. O semiproletariado transnacional (SINATTI, 2006) opóe-se a uma ordem militar que busca constranger a permanência e a sedentariedade mesmo em face de crises econômicas, catástrofes e guerras. As suas práticas antagonistas contrastam o processo global de estratificação ligada à mobilidade, construída sobre mecanismos diferenciais e privilégios reservados para as elites de san papier excelentes, que, desde sempre, gozam de liberdade de movimento em escala global: os grupos dominantes, os políticos e os managers, os artistas e os campeóes esportivos, os cientistas, os intelectuais e os religiosos.

Reatando os fios de uma história interrompida pela escravidão, pela modernização, pela industrialização e pela urbanização selvagem, as subjetividades locais marcadas por tradiçôes múltiplas têm contribuições únicas para as configuraçóes culturais e os esforços intelectuais e políticos. Várias práticas de mudança social, cultural, econômica e política, baseadas na concepção dinâmica das subjetividades que vivem articulando diferenças e mudanças, rompem o tempo linear da história (BENJAMIN, 1955) e fogem dos mecanismos fragmentários dos integralismos identitários. Buscam a emancipação de uma inclusão excludente, limitada à precária reprodução biológica de exércitos industriais de reserva (MARX, 1867) e constroem espaços para conquistar uma presença que excede as dramáticas injustiças socioeconômicas e altera as formas da participação política.

Perspectivas "por baixo" exibem a complexidade e a dinamicidade das práticas da vida cotidiana, subtraídas à lógica liminar e articuladas em arenas efervescentes nas quais distintas visóes de mundo, interesses e poderes se ligam e se contrapóem. Suas experiências emancipadoras oferecem a possibilidade de enfrentar as dramáticas desigualdades sociais e tentam superar as desastrosas abordagens assistenciais, as perigosas intervençôes emergenciais e especiais, as misericordiosas e contraditórias açôes humanitárias, em favor de iniciativas integradas e multissetoriais baseadas nas potenciais alternativas dos recursos humanos locais. Procuram formas econômicas coletivas que não favorecem um bem-estar material devastador para os laços sociais e o ambiente, rompendo com a destruição perpetuada em nome da globalização e do desenvolvimento. (ESCOBAR, 1995) 
As condiçôes dos "condenados da terra" (FANON, 1961) tornaram-se paradigmáticas como modelos para compreender as subjetividades contemporâneas, descentralizadas e deslocadas da aceleração dos mecanismos destrutivos e deslocadoras da globalização neoliberal. Posicionamentos subalternos evidenciam como os mecanismos do estado de exceção e sua fenomenologia tornaram-se elementos essenciais de todos os Estados e sérias ameaças à sua firmeza democrática: sinalizam que as características atribuídas pelo Direito romano à figura do homo sacer enquanto "outcast [...] banned man, tabooed, dangerous" (FOWLER, 1920, p. 17), ou, "incluído na ordem apenas na forma da sua exclusão" (AGAMBEN, 1995 , p. 33) não sejam mais marginalizadas numa categoria definida ou em um lugar particular, mas habitam no corpo biológico de cada ser vivo. (AGAMBEN, 1995)

Diferentes formas de subjetividade são portadoras das questôes não baseadas apenas sobre o direito de ser diferente em relação às normas hegemônicas da comunidade nacional, mas também nas concepçóes das políticas do "reconhecimento" de Charles Taylor (1994) ou da cidadania cultural de Renato Rosaldo (1993). Como alternativa aos diversos tipos de fragmentaçóes culturais e raciais, superam a aquisição formal e político-legal de princípios já definidos e reconhecem ativamente novos direitos, baseados na ligação entre aspectos materiais, econômicos, culturais, históricos, sociopsicológicos, ecológicos e políticos.

As configuraçôes do que Ong (1999) chama de "cidadania flexível”, articulações de filiações contingentes e complexas com pertencimentos e negociações múltiplas, consideram a cidadania, ao invés de uma estrutura monolítica externa em face às preocupaçóes e à vida cotidiana das pessoas, como um espaço vital (HOLSON; APADURAI, 1996) e um processo dialógico, (GRILLO; PRATT, 2006) Como tal, pode ser avaliada analisando-se as microdinâmicas de inclusão e de exclusão no dia a dia dos atores, através das quais os direitos são negociados, realizados, negados ou alterados.

As incongruências entre a cidadania formal e substantiva, bem como entre a cidadania como direito humano universal e a sua discriminação nacional, configuram a possibilidade de repensar os fundamentos da cidadania e das relações, não mais imediatas, entre Estado e Nação (HABERMAS, 1996), bem como a ligação entre os indivíduos, o Estado e as formas alternativas dos poderes, ocultos ou públicos, que intervêm na regulamentação das vidas das pessoas.

As interpretaçóes do conceito de cidadania como processo construtivo e práxis transformadora convidam a articular as lutas por direitos particulares com a mais ampla busca pela abolição dos mecanismos de produção da exclusão e pela mudança das formas de política. As superaçóes do conceito de identidade possibilitam as elaboraçóes de políticas abertas e disponíveis para as negociaçóes, as uniōes e as solidariedades. O universalismo do diálogo (BUTLER, 1995) e da 
cooperação relançam as diferenças no etos das suas interdependências. (BALIBAR, 1988) Supera às preocupantes naturalizaçóes e às perigosas politicas fragmentarias e excludentes e permite identificar objetivos e valores comuns.

\section{Referências}

AGAMBEN, G. Homo Sacer I. Il potere sovrano e la vita nuda. Torino: Einaudi, 1995. . Stato di eccezione, Homo Sacer II. Torino: Bollati Boringhieri, 2003.

AMSELLE, J. L. Branchements. Anthropologie de l'universalité des cultures. Paris: Flammarion, 2001.

ANDERSON, B. Imagined Communities. Reflections on the Origins and Spread of Nationalism. London: Verso, 1983.

APPADURAI, A. Modernity at Large. Cultural Dimensions of Globalization. Minneapolis-London: University of Minnesota Press, 1996.

. Dead Certainty. Ethnic Violence in the Era of Globalization. Public Culture, New York, v. 10, n. 2, p. 225-247, 1998.

2005.

. Sicuri da morire. La violenza nell'epoca della globalizzazione. Roma: Meltemi,

APPIAH, K. A. Is The Post in Post-Modernism the Post in Post-Colonialism? Critical Inquiry, Chicago, v. 17, n. 2, p. 336-357, 1991.

ARENDT, H. The Origins of Totalitarianism. New York: Harcourt Brace Jovanovich, 1951.

BALIBAR, E. Race, nation, class: les identités ambiguës. Paris: La Découverte, 1988.

BAUMANN, G. Contesting Culture. Cambridge: Cambridge University Press, 1996.

BENJAMIN, W. Schriften. Frankfurt am Main: Suhrkamp, Verlag, 1955.

BHABHA, H. K. The Location of Culture. London: Routledge, 1994.

BOWMAN, G. The Power of Violence in Identity. In: B. E. SCHMIDT; I. W. SCHRODER. (Eds.). Anthropology of Violence and Conflict. London: Routledge, 2001.

BRAIDOTTI, R. Nuovi Soggetti Nomadi. Roma: Luca Sossella Editore, 2002.

BUTLER J. Contingent Foundations: feminism and the question of postmodernism. In: BENHABIB, S. et al. Feminist Contentions: a philosophical exchange. New York: Routledge, 1995pp. 127-143.

CANCLINI, G. N. Culturas Hibridas: estrategias para entrar y salir de la modernidad. Bogotá: Banco de la Republica, 1990.

CARVAlHO, J. M. Os bestializados. São Paulo: Companhias das Letras, 1991.

CLIFFORD, J. The Predicament of Culture. Twentieth-Century Ethnography, Literature, Art. Berkeley: University of California Press, 1988. 
. Routes. Harvard: Harvard Universiาty Press, 1997.

COERTZE, C. Volkekunde. South African Journal of Ethnology, Pretoria, n. 1, p. 2-34, 1978.

COHEN, A. Two-Dimensional Man. London: Routledge \& Kegan, 1974.

COMAROFF, J.; COMAROFF, J. Modernity and its Malcontents: Ritual and Power in post-colonial Africa. Chicago: University of Chicago Press, 1993.

DAMATTA, R. Do You Know Who You are Talking To? The distinction between individual and person in Brazil. In: DAMATTA, R. (Ed.). Carnivals. Rogues and Heroes: an interpretation of the Brazilian dilemma. Notre Dame: University of Notre Dame Press, 1991, p. 137-197.

DOUGLAS, M. Purity and danger. An analysis of concepts of pollution and taboo. Harmondsworth: Penguin Books, 1970.

ERIKSON, E. H. Ontogeny of ritualization in man. Philos. Trans Roy. Soc., London, Séries B, n. 251, 1966.

ESCOBAR, A. Imagining a Post-development Era? Critical Thought, Development and Social Movements. In: J. CRUSH. Power of development. London: Routledge, 1995. p. 211-227.

FANON, F. Les Damnés de la Terre. Paris: La Découverte, 1961.

FERGUSSON, J. The Anti-Politics Machine: "Development”, Depolitization and Bureaucratic Power in Lesotho. Minneapolis: University of Minnesota Press, 1990.

FOUCAULT, M. La Volonté de savoir. Paris: Editions Gallimard, 1976.

FOWLER, W. Roman essays and interpretations. Oxford: Oxford University Press, 1920.

GADAMER, H.G. Wahreit un Methode. Tubingen: J.C.B. Mohr, 1965.

GILROY, P. The Black Atlantic. Modernity and Double-Consciuosness. London: Harvard University Press, 1993.

GOUREVITCH, P. We wish to inform you that tomorrow we will be killed with our families: stories from Rwanda. New York: Farrar, Status and Giroux, 1998.

GRILLO R.; PRATT J. The Politics of Recognizing Difference: Multiculturtalism Italian-Style. Farnham: Ashgate Publishing, 2002.

HABERMAS, J. Kampf um Anerkennung im demokratischen Rechtsstaat. In: HABERMAS, J.; TAYLOR, C. Einbeziehung des Anderen. Frankfurt am Main: Suhrkamp, 1996. p. 237-276.

HALL, S. Authoritarian Populism: a Reply to Jessop et al. New Left Review, London, v. 151, p. 115-124, may-june 1985.

HAYDEN, R. M. Imagined communities and real victims: Self-determination and ethnic cleansing in Yugoslavia. American Anthropologist, Arlington, v. 23, n. 4, p. 783-801, 1996.

HEGEL, G. W. F. Die Phänomenologie des Geistes. Hamburg: Meiner Verlag, 1807. 
HOLSON, J.; APPADURAI, A. Cities and Citizenship. Public Cultures, Durham, n. 4, v. 8, p. 187-204, 1996.

HOLSTON, J.; CALDEIRA, T. Democracy, Law and Violence: Disjunctions of Brazilian Citizenship. In: AGUERO, F.; STARK, J. (Eds.). Fault Lines of Democracy in Post-Transition Latin America. Miami: University of Miami, 1998, p. 263-296.

LAUTIER, B. Os amores tumulados entre o Estado e a economia informal. Contemporaneidade e Educação, Rio de Janeiro, n. 1, v. II, p. 23-29, 1997.

MACHADO DA SILVA, L. A. A Continuidade do Problema de Favela. In: LIPPI OLIVEIRA, L. (Org.). Cidade: História e Desafios. Rio de Janeiro: Fundação Getúlio Vargas, 2002, p. 220-237.

MALIGHETTI, R. (Org.). Antropologia Applicata. Dal nativo che cambia al mondi ibrido. Milano: Edizioni Unicopli, 2001.

. (Org.). Oltre lo sviluppo. Le prospettive dell'antropologia. Roma: Meltemi, 2005.

. (Org.). Politiche dellidentità. Roma: Meltemi, 2007.

. Biopolitiche di eccezione e pratiche di resistenza. Confluenze: Rivista di Studi Iberoamericani, Bologna, v. 3, n. 2, p. 22-34, 2011.

MARX, K. The Revolt in the Indian Army. New York Daily Tribune, New York, jul. 15, 1857.

. Das Kapital: kritik der politishen ekonomie. Berlin: Dietz, 1867.

ONG, A. Flexible Citizenship. The Cultural Logic of Transnationality. Durham and London: Duke University Press, 1999.

RABINOW, P. Anthropos Today: reflections on modern equipment. Princeton: University Press, 2003.

REMOTTI F. L'ossesione Identitaria. Roma-Bari: Laterza, 2010.

ROSALDO, R. Culture and Truth. Boston: Beacon Press, 1993.

ROUSE, R. Questions of identity, personhood and collectivity in transnational migration to the United States. Critique of Anthropology, London, v. 15, n. 4, p. 351-380, 1995.

SAID, E. Culture and Imperialism. London: Vintage, 1993.

SALES, T. Raízes da desigualdade social na cultura brasileira. Revista Brasileira de Ciências Sociais, São Paulo/Anpocs, v. 9, n. 25, p. 26-37, 1994.

SANTOS, W. G. Cidadania e Justiça. Rio de Janeiro: Campus, 1979.

SAYAD, A. L'immigration ou les paradoxes de l'altérité. Paris: Eds. Universitaires, 1991.

SCHMITT, C. Politische Theologie, Vier Kapitel zur Lehre von der Souveränität. Munchen-Leipzig: Dunkler \&. Humblot, 1922. 
SINATTI, G. Diasporic cosmopolitanism and conservative translocalism: narratives of nation among Senegalese migrants in Italy. Studies in Ethnicity and Nationalism, London, v. 6, n. 3, p. 29-50, 2006.

SOUTHALL, A. The Illusion of Tribe. Journal of Asian and African Studies, London, v. 5, p. 28-50, 1970.

SPIVAK, G. A Critique of Post-Colonial Reason: Towards a History of the Vanishing Present. Harvard: Harvard University Press, 1999.

TAGUIEFF, P. A. La force du préjugé. Essai sur le racisme et des doubles. Paris: Editions La Découverte, 1988.

TAYLOR, C. Multiculturalism: examining the politics of recognition. Princeton: Princeton University Press, 1994.

VENTURA, Z. Cidade Partida. São Paulo: Companhia das Letras, 1994.

WERNECK, V. L. A Revolução Passiva. Iberismo e Americanismo no Brasil. Rio de Janeiro: Revan, 1997.

Recebido em 28 de novembro de 2013.

Aprovado em 24 de outubro de 2014. 